\title{
The Astrophysical Plasmadynamic Explorer (APEX): A High Resolution Spectroscopic Observatory
}

M.P.Kowalski, et al.

This article was submitted to

Astronomical Telescopes and Instrumentation, Waikoloa, Hawaii, August 22-28, 2002

U.S. Department of Energy

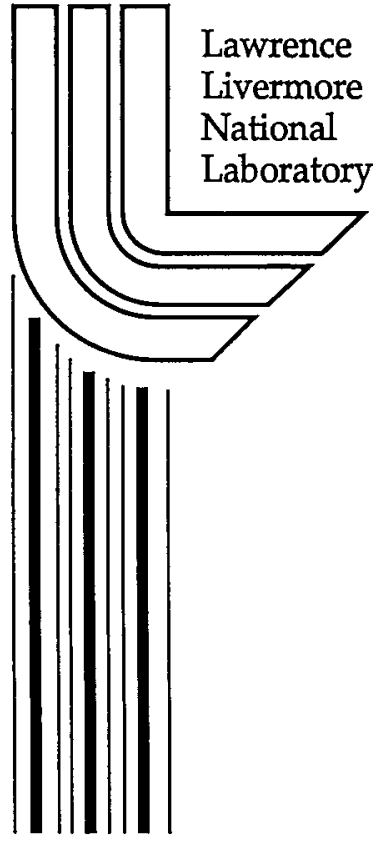

July 18, 2002 


\section{DISCLAIMER}

This document was prepared as an account of work sponsored by an agency of the United States Government. Neither the United States Government nor the University of California nor any of their employees, makes any warranty, express or implied, or assumes any legal liability or responsibility for the accuracy, completeness, or usefulness of any information, apparatus, product, or process disclosed, or represents that its use would not infringe privately owned rights. Reference herein to any specific commercial product, process, or service by trade name, trademark, manufacturer, or otherwise, does not necessarily constitute or imply its endorsement, recommendation, or favoring by the United States Government or the University of California. The views and opinions of authors expressed herein do not necessarily state or reflect those of the United States Government or the University of California, and shall not be used for advertising or product endorsement purposes.

This is a preprint of a paper intended for publication in a journal or proceedings. Since changes may be made before publication, this preprint is made available with the understanding that it will not be cited or reproduced without the permission of the author.

This report has been reproduced directly from the best available copy.

Available electronically at http://www.doe.gov/bridge

Available for a processing fee to U.S. Department of Energy and its contractors in paper from

U.S. Department of Energy

Office of Scientific and Technical Information

P.O. Box 62

Oak Ridge, TN 37831-0062

Telephone: (865) 576-8401

Facsimile: (865) 576-5728

E-mail: reports@adonis.osti.gov

Available for the sale to the public from

U.S. Department of Commerce

National Technical Information Service

5285 Port Royal Road

Springfield, VA 22161

Telephone: (800) 553-6847

Facsimile: (703) 605-6900

E-mail: orders@ntis.fedworld.gov

Online ordering: http://www.ntis.gov/ordering.htm

OR

Lawrence Livermore National Laboratory

Technical Information Department's Digital Library

http://www.llnl.gov/tid/Library.html 


\title{
The Astrophysical Plasmadynamic Explorer (APEX): A High Resolution Spectroscopic Observatory
}

\author{
M. P. Kowalskia , R. G. Cruddace ${ }^{a}$, K. S. Wood, D. J. Yentis ${ }^{a}$, H. Gursky ${ }^{a}$, \\ T. W. Barbee ${ }^{b}$, Jr., W. H. Goldstein ${ }^{b}$, J. F. Kordas ${ }^{b}$, \\ G. G. Fritz ${ }^{\mathrm{c}}$, W. R. Hunter ${ }^{\mathrm{d}}$, \\ M. A. Barstow, N. P. Bannister ${ }^{e}$, \\ J. L. Culhane ${ }^{f}$, J. S. Lapington ${ }^{\text {f,g }}$ \\ ${ }^{2}$ Naval Research Laboratory, Code 7655, Washington, DC 20375 \\ ${ }^{b}$ Lawrence Livermore National Laboratory, 7000 East Ave., P.O. Box 808, Livermore, CA 94550 \\ ${ }^{c}$ PRAXIS, Inc., 2200 Mill Road $5^{\text {th }}$ Floor, Alexandria, VA 22314 \\ ${ }^{\text {d}}$ SFA Inc., 9315 Largo. Dr. West, Suite 200, Largo, MD 20774 \\ ${ }^{\mathrm{e}}$ University of Leicester, Leicester LE1 7RH, England \\ fMullard Space Science Laboratory, Holmbury St. Mary, Dorking, Surrey RH5WNT, England \\ ${ }^{g}$ Boston University. CAS Space Physics, 725 Commonwealth Ave., Boston, MA 02215
}

\begin{abstract}
EUVE and the ROSAT WFC have left a tremendous legacy in astrophysics at EUV wavelengths. More recently, Chandra and $X M M$-Newton have demonstrated at X-ray wavelengths the power of high-resolution astronomical spectroscopy, which allows the identification of weak emission lines, the measurement of Doppler shifts and line profiles, and the detection of narrow absorption features. This leads to a complete understanding of the density, temperature, abundance, magnetic, and dynamic structure of astrophysical plasmas. However, the termination of the $E U V E$ mission has left a gaping hole in spectral coverage at crucial EUV wavelengths $(\sim 100-300 \AA)$, where hot $\left(10^{5}-10^{8} \mathrm{~K}\right)$ plasmas radiate most strongly and produce critical spectral diagnostics. CHIPS will fill this hole only partially as it is optimized for diffuse emission and has only moderate resolution ( $\mathrm{R} \sim 150)$. For discrete sources, we have successfully flown a follow-on instrument to the EUVE spectrometer $\left(\mathrm{A}_{\mathrm{eff}} \sim 1 \mathrm{~cm}^{2}, \mathrm{R} \sim 400\right)$, the high-resolution spectrometer $J-P E X\left(\mathrm{~A}_{\mathrm{eff}} \sim 3 \mathrm{~cm}^{2}, \mathrm{R} \sim 3000\right)$. Here we build on the $J-$ $P E X$ prototype and present a strawman design for an orbiting spectroscopic observatory, APEX, a SMEX-class instrument containing a suite of 8 spectrometers that together achieve both high effective area $\left(A_{\text {eff }}>20 \mathrm{~cm}^{2}\right)$ and high spectral resolution $(R \sim 10,000)$ over the range 100-300 $\AA$. We also discuss alternate configurations for shorter and longer wavelengths.
\end{abstract}

Keywords: Satellite Instruments, Spectrometers, Diffraction gratings, Multilayer coatings, Extreme Ultraviolet

\section{INTRODUCTION}

Spectroscopy has evolved into an indispensable tool in the task of measuring the composition, density, temperature, velocity and magnetic field of astrophysical plasmas. Now EUVE, Chandra and XMM-Newton have moved astronomical spectroscopy into the EUV and X-ray wavebands, where hot $\left(10^{5}-10^{8} \mathrm{~K}\right)$ plasmas radiate most strongly. However, further developments are needed to exploit the full range of plasma diagnostic techniques developed in laboratory and solar physics, including instruments of higher resolution, in particular spectrometers which could, for example, resolve and measure spectral lines unambiguously and measure line profiles and Doppler shifts. A desirable goal is a resolving power $R>10,000$ $C=0.01$ at 100 , or $\left.V=30 \mathrm{~km} \mathrm{~s}^{-1}\right)$. The EUV waveband between 100 and 300 is most promising for high-resolution spectroscopy of hot plasmas, for the following reasons:

(1) The interstellar medium (ISM) has relatively low opacity out to distances of order $100 \mathrm{pc}$ and is patchy, allowing diverse galactic studies and some extragalactic observations.

(2) There are many strong spectral lines produced by plasmas at temperatures between $2 \times 10^{5}$ and $2 \times 10^{7} \mathrm{~K} .{ }^{1}$

(3) Multilayer-coated, normal-incidence optics may be used. They have smaller aberrations than grazing-incidence optics, promise large collecting area, and allow designs with a single optical element.

In this paper we present a strawman design for an orbiting high-resolution EUV spectroscopic observatory, $A P E X$, the Astrophysical Plasmadynamic EXplorer, a SMEX (SMall EXplorer) class instrument. 


\section{SCIENCE JUSTIFICATION}

It is difficult to predict the course of EUV astronomy following the EUVE and ROSAT Wide Field Camera (WFC) missions, but in regard to high-resolution spectroscopy we give as examples four research areas with exciting prospects. The first is the formation and characteristics of hot coronae around pre-main-sequence (PMS) stars, stars on the main sequence (MS), subgiants and the hotter giant stars. The driving force is the magnetic dynamo in stellar convection zones, which is most active and volatile when a star is young and in the PMS stage. There are violent eruptions of field from the star, which may play an important role as planetary systems start to evolve. The activity diminishes after the star enters the MS, but our sun shows how complex this activity is and how it influences conditions around the planets. Thus at some point searches for life in other planetary systems must take into account the influence of the stellar corona. When the sun is compared to more active stars, where the energy released may be up to $10^{4}$ times that in solar events, the difficulties of understanding stellar dynamos as a galactic phenomenon become apparent. The study of stellar dynamos is bound to the study of the distribution, temperature, density, composition and dynamics of the hot coronal plasma, and of the origin of active regions, loops and flares. Solar research has demonstrated the importance of high-resolution spectroscopy in such research.

The second area is to understand how stars evolve from the giant branch into the white dwarf (WD) region of the H-R diagram. The formation and evolution of WD stars is a major facet of galactic evolution, and their distribution and characteristics are important in estimating the age and mass of the galaxy. The third area is the study of accretion in cataclysmic variable (CV) systems. For example, the large width of some AM Her spectral lines implies that turbulence or Zeeman splitting is at work in the accretion flow, and to clarify the accretion disk behavior during dwarf novae outbursts the time-dependence of selected lines needs to be established. Both tasks require high-resolution spectroscopy. The fourth area is the study of $\mathrm{He}$ in the local interstellar medium (LISM) by making high-resolution measurements of HeII absorption lines in WD spectra. Helium is the element most sensitive to non-equilibrium ionization conditions, and is a sensitive indicator of ionizing sources in the LISM. HeII and HeI measurements could test whether the gas in the LISM is in an ionization recombination phase following a supernova a few million years ago. This would be an important adjunct to the CHIIS mission to survey the structure, ionization state and cooling processes of the $10^{6} \mathrm{~K}$ gas in the LISM. The table below shows the wide variety of targets relating to these areas that would be covered by $A P E X$.

Table 1. Extracts from an Extensive APEX Target List

\begin{tabular}{|c|c|c|c|c|c|}
\hline Tanget & $\begin{array}{l}5 \\
\frac{5}{7} \\
8 \\
4\end{array}$ & $\frac{8}{\frac{8}{8}}$ & 3 & 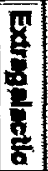 & Comments \\
\hline GD659 & $\mathbf{x}$ & & & & Purs H, Fill, SU Detection with EUVE, prime ISU target \\
\hline FEKE 246 & $\bar{x}$ & & & & 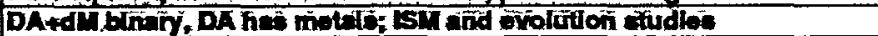 \\
\hline GTसक्2 & $\ddot{\bar{x}}$ & & & & 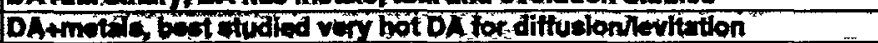 \\
\hline GD 7 & $x$ & & & & Purs $\mathrm{H}_{\mathrm{x}}$ low nH, prime isM torget \\
\hline Siptus B & $\underline{x}$ & & & & Nesreat wD, mascalve and important for evolution and $\mathrm{SW}$ \\
\hline PGT123+18 & $\bar{x}$ & & & & 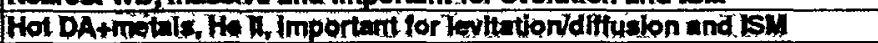 \\
\hline EG 187 & $\frac{x}{x}$ & & & & Intermodiate tomp Da, low nH, prime isM tanget \\
\hline HZQ3 & $x$ & & & & Archetypal hot DA, low nH, prime ISU target and cal source \\
\hline AEV2156-643 & $\bar{x}$ & & & & 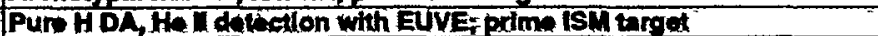 \\
\hline REV 2214491 & $x$ & & & & ? Hottest DA+motals, extrome ceac for Jevitationdiffusion \\
\hline V471 Tnu & $\mathbf{x}$ & $\mathbf{X}$ & & & Pro-CV; K2V+DA, $p=0.022 d$ \\
\hline Wrepp & & & $\bar{x}$ & & 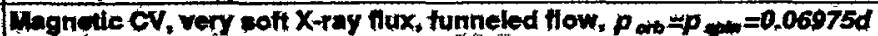 \\
\hline ÚGan & & & $\bar{x}$ & & Non-magnetic CV (drant nova) whit $X$-ray outbursts, $p=0.177 d$ \\
\hline SScyg & & & 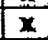 & & 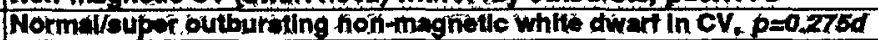 \\
\hline Procyon & & $\bar{x}$ & & & Thin convection zone solar-like getivity \\
\hline$\alpha \operatorname{con} A B$ & & $\bar{x}$ & & & 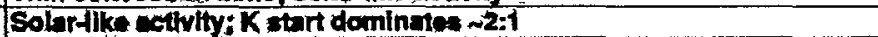 \\
\hline Proxcon & & $\bar{x}$ & & & EUV -briahtes "low" activity late M dwart \\
\hline AUTtic & & $\bar{x}$ & & & Very active I dwant \\
\hline$\beta C A$ & & $\bar{x}$ & & & First wo or Clump glant \\
\hline ABDor & & $\bar{x}$ & & & Prows, $p=0.51 d$ \\
\hline a Aur & & $x$ & & & RS CVn Asychronous binary; Hortzsprung gap + clump? \\
\hline ARLEC & & $\mathbf{x}$ & & & AS CVn p $=200$, loft degreses, ecllpsing \\
\hline VS Cep & & $x$ & & & W Uma contact blinary: $p=0.28 d$ \\
\hline resm & & $\bar{x}$ & & & 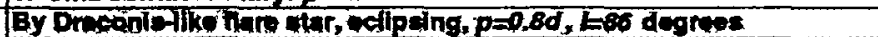 \\
\hline PKS 2165-304 & & & & $\mathbf{x}$ & BL L w the ojbet $(z=0,17)$ \\
\hline
\end{tabular}


These four areas are also part of the NASA major theme "Structure and Evolution of the Universe", and pursue Objective 3 of the NASA Strategic Plan for Space Science. However the first area has a broader context and is linked to the other major themes, "The Sun-Earth Connection" and "Astronomical Search for Origins", and to Objectives 7 ("Understand our changing Sun and its effects throughout the Solar System") and 4 ("Look for signs of life in other planetary.systems") of the NASA Strategic Plan.

To illustrate the power of high-resolution EUV spectroscopy we developed an instrument (R 3000; effective area $\left.A_{\text {eff }} \sim 3 \mathrm{~cm}^{2}\right)$ called J-PEX (Joint astrophysical plasmadynamic Experiment) that was launched on 21 February 2001 by the NASA sounding rocket 36.195 DG and observed the white dwarf G191-B2B successfully. ${ }^{2-5}$ The goal was to detect and measure absorption features of singly ionized helium and heavier elements in the wavelength region $220-245 \AA$. The study investigated the relative amounts of photospheric and interstellar helium, a crucial step in understanding the evolutionary paths of white dwarf stars.

Fig. 1 compares the observed spectrum (black error bars) with model predictions (red histogram) based on a homogeneous composition stellar atmosphere and including interstellar $\mathrm{HI}, \mathrm{HeI}$ and $\mathrm{HeII}$ absorption. The $\mathrm{HI}$ and HeI column densities were fixed at values obtained from analysis of the broader band, lower resolution EUVE spectrum, and the values of $\mathrm{T}$ and $\mathrm{g}$ were taken from the latest optical analysis $(T=54,000 \mathrm{~K}, \log \mathrm{g}=7.5){ }^{6,7}$ The interstellar HeIl column density and photospheric He abundance were allowed to vary freely, and the best match to the model was obtained by minimizing a Cash statistic. The best-fit model folded through the $J-P E X$ instrument response assumed a resolving power of $4000(0.057$ FWHM), and the data's $\mathrm{S} / \mathrm{N}$ averaged 5.0 per sampling bin. The observed spectrum shows a continuum modulated by numerous absorption lines and by absorption in the ISM. The good agreement between the model and the data is striking, with the most prominent resolved feature a strong OIV absorption line at $233.5 \AA$. Between 227 and 232 _ is a broad "bump", a characteristic of the overlapping series of interstellar Hell absorption lines superposed on a continuum. Taken together with the strong depression of the flux below 227 , this is conclusive proof that interstellar or circumstellar HeII is present in the line of sight. The strongest expected photospheric line at $243 \AA$ was too close to the noise level to claim a direct detection of photospheric helium as yet. However, the best fit was obtained for $\mathrm{N}_{\mathrm{Hell}}=5.97 \times 10^{17} \mathrm{~cm}^{-2}$ and $\mathrm{He} / \mathrm{H}=1.6 \times 10^{-5}$, and the joint confidence contour for these parameters produced $99 \%$ confidence limits of $(5.76-6.18) \times 10^{17} \mathrm{~cm}^{-2}$ for $\mathrm{N}_{\text {HeII }}$ and (1.31-1.91) $\times 10^{-5}$ for $\mathrm{He} / \mathrm{H}$.

Further analysis is in progress including fits with stratified models. In a companion paper in this conference, we have examined the effect of including another component of ISM HeII separated in velocity from that of the local interstellar cloud. ${ }^{3}$ This action significantly improves the fit, produces a velocity difference of $22 \mathrm{~km} \mathrm{~s}^{-1}$ between the two ISM components, and may reduce the implied He ionization fraction to values more consistent with other observations. Hence the ability to obtain dynamical information, even in the short $J-P E X$ sounding rocket flight, has profound implications for the models.

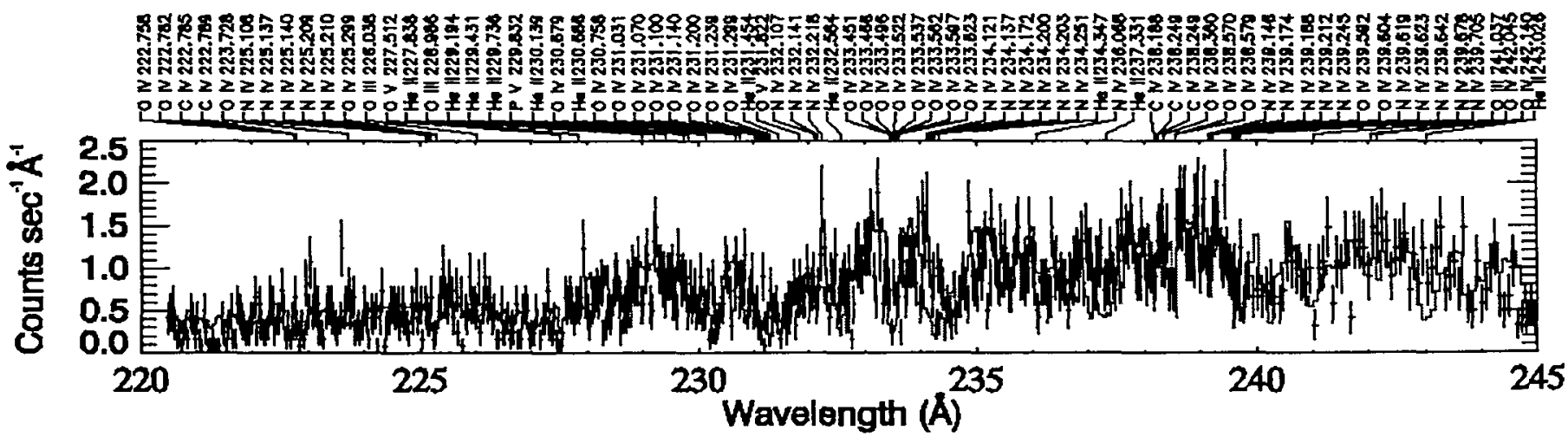

Fig. 1. High-resolution spectrum of G191-B2B obtained by the J-PEX spectrometer in the band $220-245 \AA$ (error bars). The red histogram is the best-fit model of the stellar photosphere and the ISM (see text). The strongest predicted lines of $\mathrm{He}, \mathrm{C}, \mathrm{N}, \mathrm{O}$, and $\mathrm{P}$ are labeled by ionization state and wavelength. Fe and $\mathrm{Ni}$ lines, too numerous to list, produce the unlabelled features and the broader absorption structures. 


\section{APEX STRAWMAN DESIGN}

The J-PEX sounding rocket payload had two advantages, high $\mathrm{A}_{\mathrm{eff}}$ and $\mathrm{R}$, and two disadvantages, a limited bandwidth and limited observation time. Specific scientific questions can be addressed effectively using sounding rockets, but studying the large and diverse samples revealed by EUVE and the ROSAT WFC requires the long observation times provided by an orbiting observatory. Moreover, a SMEX satellite affords much more volume than a sounding rocket so that the bandwidth problem may be overcome by using several spectrometers, each optimized for a different waveband. APEX fits comfortably within the Taurus 2210 payload shroud of a SMEX (Fig. 2) and contains no moving parts (other than the payload door) hence minimizing risk.

Fig. 3 shows the instrument aperture. APEX contains a suite of 8 spectrometers, each having a grating, a collimator, and a detector. Each spectrometer is mounted on an optical bench, which is isolated from thermal and vibrational loads in the payload structure. The optical bench also provides independent capability to align and focus the gratings during calibration and flight. The aperture end of the payload contains the collimators, the detectors, instrument support electronics (not shown), the payload door (not shown), and the star tracker used in acquiring targets (not shown). The opposite end contains the gratings and other payload support system (payload command and control electronics, batteries, ACS, telemetry, etc.). Unfolding solar cell panels would reside before launch in the annular volume between the APEX outer skin and the maximum payload envelope. The honeycomb collimator modules restrict the field of view $\left(\sim 1^{\circ}\right)$ to limit the diffuse EUV and UV radiation that enters the spectrometers, and $J-P E X$ has provided us with much experience in designing and fabricating suitable modules. ${ }^{9}$ This radiation is further attenuated to acceptable levels using a thin-film filter mounted inside each detector vacuum enclosure slightly upstream of its photocathode. (Thus the filter suffers no acoustic loads during T\&E and launch.) $J-P E X$ used an aluminum filter mounted on a nickel mesh, and suitable materials are available for all the wavebands discussed in this paper. Internal baffles (not shown) trap and attenuate unwanted diffracted orders, zero order, and scattered light from the gratings. Because sounding rocket observation times are short, J-PEX was launched under vacuum to ensure adequate time for outgassing. No such requirement exists for a satellite instrument; however, it may be desirable to make the payload door a vacuum seal (or doors for each spectrometer) for ease in handling during calibration. During idle periods the payload would be backfilled with an inert gas to retard contamination and degradation of the multilayer-coated optics.

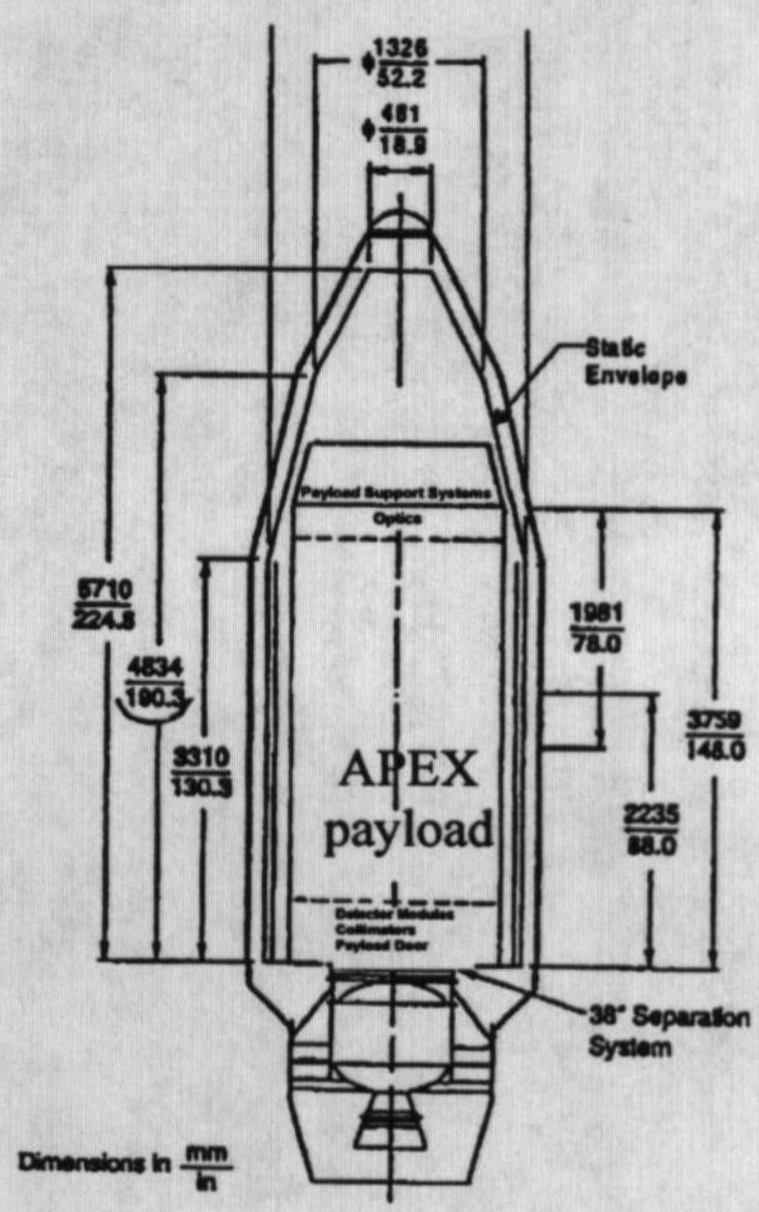

Fig. 2. APEX payload in Taurus 2210 shroud.

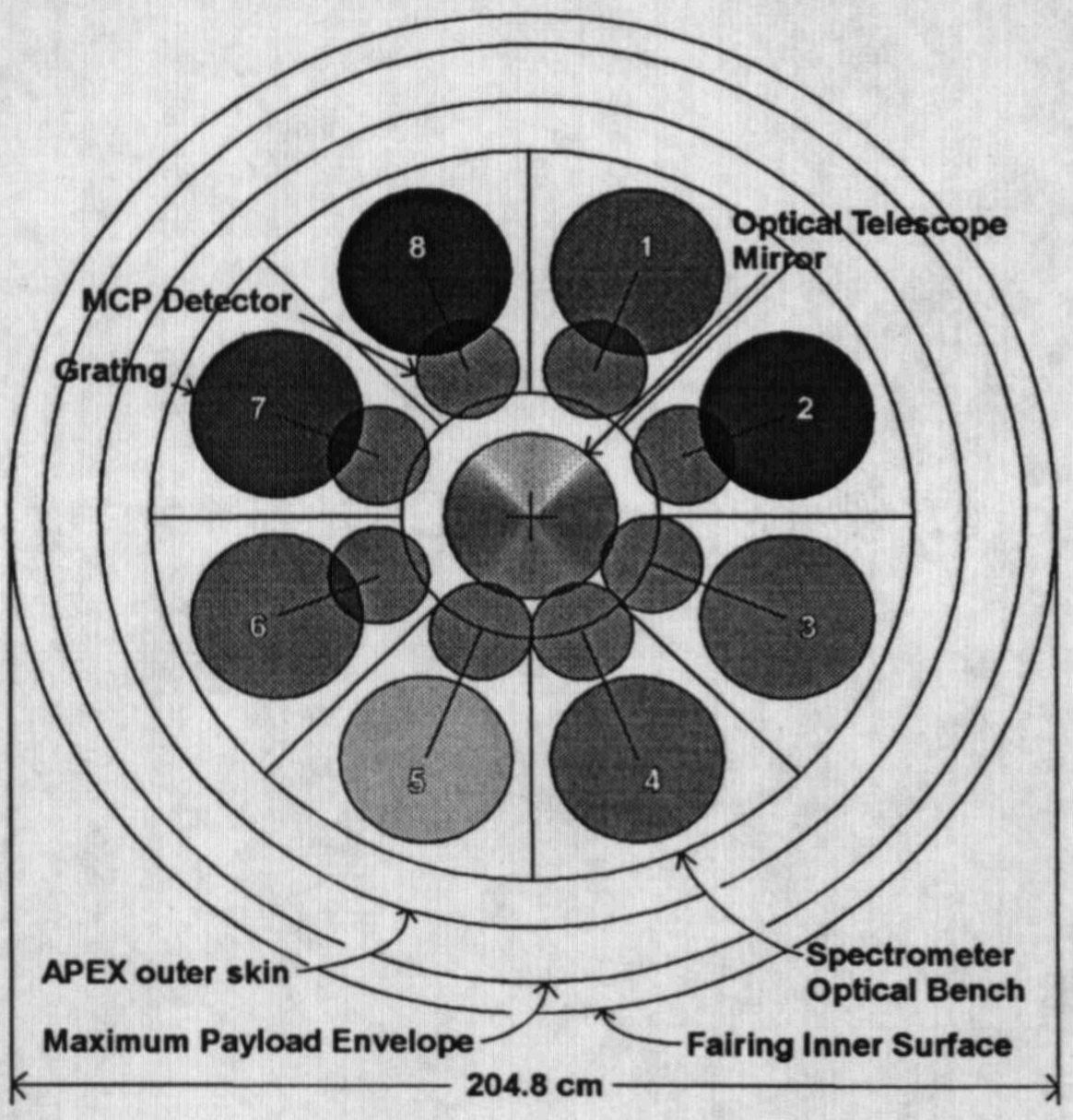

Fig. 3. APEX Instrument Aperture.

Each microchannel plate (MCP) detector would have its own vacuum enclosure to allow operation independent of the spectrometer. For simplicity a circle of diameter $200 \mathrm{~mm}$ was chosen as the detector footprint for this strawman payload. However, the size and shape of these enclosures is under study, and therefore the slight obscuration of the grating by the detector in some of the spectrometers (Fig. 3) should not be taken seriously. Each detector would have multiple MCPs (e.g., chevron or Z-stack configuration) to produce high gain in saturation mode with low background noise. Both high quantum 
efficiency $(\mathrm{QE})$ and high spatial resolution, especially in the dispersion direction (the line shown in projection that connects the grating and detector centers), are essential for sensitive high-resolution spectroscopy. At EUV wavelengths, QE is dominated by the photoelectric yield of the top MCP and its photocathode. Common choices of photocathodes include CsI and $\mathrm{KBr}$. Spatial resolution is determined by the pore spacing of the MCPs and by the charge collection anode. Glass MCPs produced from boules using heating and drawing techniques have been the standard for many years, and channel diameters of $12.5 \mathrm{~m}$ and more recently $6 \mathrm{~m}$ are available. $J-P E X$ used a chevron configuration of glass MCPs with a CsI photocathode deposited on the top MCP. However, current research with silicon MCPs has produced results that promise to surpass the performance of glass MCPs without encountering any of their problems, and these may be considered for use in $A P E X{ }^{10} \mathrm{~A}$ number of anodes are available that produce high-resolution images including the vernier anode, ${ }^{11}$ delay-line anodes, ${ }^{10}$ and cross-strip anodes. ${ }^{10}$ The $J$-PEX MCP detector used a vernier anode that produced high linearity with a resolution less than 20 _m, which is adequate for $A P E X$. However, further development with all these anodes has been very successful, leading to images of 6 _m MCP pores.

The figured gratings are all mounted in a Wadsworth configuration operating at near-normal incidence. They have a focal length of $3 \mathrm{~m}$ and a diameter of $340 \mathrm{~mm}$. With allowance for the footprint and volume of support subsystems no more than 8 such gratings can fit within a payload designed for the Taurus 2201 shroud. Grating details are given in the following sections. As with $J-P E X$, the $A P E X$ payload also contains a telescope operating at optical/UV wavelengths. The telescope mirror is $\mathbf{3 4 0} \mathrm{mm}$ in diameter, and a CCD camera (not shown) is mounted at its prime focus. This telescope is co-aligned to the spectrometers and views the target and/or adjacent stars. Its purpose is to provide an accurate aspect measurement while on target. Each spectral photon is time-tagged and interpolation between CCD images produces an accurate measurement of the instantaneous pointing direction when the photon was recorded. Thus $A P E X$ does not require high pointing accuracy, only that payload jitter rates be small enough so that the CCD images can be interpolated accurately. This type of telescope proved highly successful on the J-PEX mission, and the larger $A P E X$ mirror provides more sensitive measurements (the $J$ $P E X$ mirror was only $135 \mathrm{~mm}$ in diameter).

\section{EFFECTIVE AREA FOR APEX BASELINE CONFIGURATION}

The EUV flux from all astrophysical objects other than the sun is relatively weak. Although observation times in a satellite may be as large as needed, many of the APEX targets vary on dynamical time-scales of hours to days. Thus high $\mathrm{A}_{\text {efr }}$ is necessary to produce temporally resolved high-resolution spectra. The simple $A P E X$ optical design means that the effective area of each spectrometer is the product of only 5 factors: the transmittance of the collimator, the geometrical area of the grating, the efficiency of the grating, the transmittance of the filter, and the $\mathrm{QE}$ of the MCP detector. It is beyond the scope of this paper to investigate all these factors in detail, but the goal is to maximize all them. Little can be done to improve

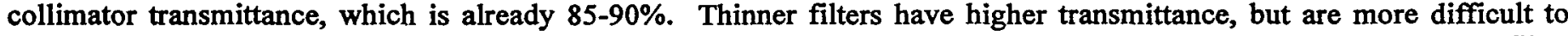
fabricate without pinholes, are more fragile, and transmit more unwanted diffuse EUV/UV radiation. Therefore, filter thickness and ultimately transmission is a tradeoff among these competing requirements. In the following calculations of effective area, filter transmittances were derived using on-line software available at the Center for X-ray Optics website (http://www-cxro.lbl.gov/). We assumed all filters were mounted on a mesh with $85 \%$ transmittance. MCP detector QE values were taken from the literature and at EUV wavelengths are in the range $15-60 \%$. Collimator transmittance was assumed to be $90 \%$. In the remainder of this paper we concentrate on grating issues (area and efficiency).

For over a decade we have pursued the goal of achieving high efficiency in a normal-incidence instrument by using ultrasmooth holographic ion-etched gratings that have been coated with high-reflectance multilayers. ${ }^{12}$ We have measured efficiencies in the range $5-16 \%$ with both laminar and blazed gratings at wavelengths between 100 and $300 \AA,{ }^{13-17}$ and in the laminar case the groove efficiency of the underlying grating substrate now approaches the theoretical limit for the first order of $40.5 \% .^{18}$ For the scalar regime in which we operate, the measured grating efficiency is the product of the grating groove efficiency and the reflectance of the coating (whether a multilayer, single layer, or bare surface), and so there are essentially 6 factors in calculating effective area. Moreover, our investigations have produced excellent agreement between theoretical models and measurements for multilayers, ${ }^{13-17}$ bare ion-etched gratings, ${ }^{1,20}$ and multilayer gratings. ${ }^{13-17}$ Finally, we have shown that the dispersive characteristics of gratings are unchanged with the application of the multilayer, and in one case a diffraction-limited resolving power of 14,000 was achieved. ${ }^{21}$

All these research results were obtained using relatively small $(\sim 25 \mathrm{~mm}$ diameter $)$ sample gratings. However, $J-P E X$ contained four large $(160 \times 90 \mathrm{~mm})$ identical spherical laminar gratings, coated with identical multilayers, and mounted in a off-axis Wadsworth configuration. Progress has been steady at a number of commercial vendors (e.g., Zeiss, Jobin-Yvon) in the fabrication of large area gratings. High-quality ion-etched gratings of diameter $340 \mathrm{~mm}$ are now feasible and we have 
baselined them for APEX. Systems for the deposition of high-reflectance multilayers uniformly over large areas have been available for several years now at the Lawrence Livermore National Laboratory (e.g., J-PEX, TRACE) and elsewhere. Multilayer technology is also backed by accurate theoretical models and a vast database of measured material properties.

As part of the $J-P E X$ calibration effort we mapped the efficiency of the gratings after multilayer coating at the Naval Research Laboratory beamline X-24C at the National Synchrotron Light Source, Brookhaven National Laboratory. ${ }^{22,23}$ This facility consists of a grating-crystal monochromator, ${ }^{24,25}$ to disperse the synchrotron light, and a number of selectable thinfilm filters, which suppress high-order radiation from the monochromator and provide absorption edges for wavelength calibration. The $J-P E X$ gratings were calibrated in a reflectometer, ${ }^{26}$ and a recent upgrade to this facility allows for the calibration of $A P E X$-sized gratings. For J-PEX, the average measured peak efficiency (@ $234 \AA$ ) ranged from 10.3 $\pm 0.6 \%$ for the best grating to $7.2 \pm 1.2 \%$ for the worst. (Problems with cleaning procedures, since remedied, reduced the efficiency of two gratings.) The quoted uncertainty is dominated by the standard deviation in the number of measurements ( 20$)$, and may be taken as a measure of uniformity, which was less than $10 \%$ for the best gratings. The instrumental uncertainties are small in both the measured efficiency (1-3\% of the measured value) and the wavelength scale $(0.3 \AA),{ }^{27}$ giving us strong confidence that $A P E X$ could be calibrated accurately.

The most efficient $J-P E X$ gratings had groove efficiencies in the range $31-34 \%$, approaching the theoretical limit of $40.5 \%$ for a laminar grating used in the first inside order. However, blazed gratings offer two distinct advantages over laminar gratings. First, the groove efficiency of an ideal blazed grating can approach $100 \%$. Second, this performance may be achieved in higher grating orders, allowing the groove density to be held within practical bounds at the shorter wavelengths or in applications demanding higher resolving power. However, to date difficulties in manufacturing an ideal blazed groove profile of low roughness at the low blaze angles required for EUV applications $\left(\sim 1^{\circ}-4^{\circ}\right)$ have limited groove efficiencies to less than 27\%. Atomic force microscopy (AFM) showed the reason to be rounding of the ideal blaze (sawtooth) groove profile during ion-etching, when a high groove density was combined with the desired low blaze angle. A process developed at Zeiss ${ }^{28}$ corrects this effect significantly. An ion-etched grating with a relatively high blaze angle $\left(-6^{\circ}-10^{\circ}\right)$ is immersed in a liquid polymer, withdrawn parallel to the grooves, and then baked. The liquid in the grooves assumes a shape, which modifies the blaze angle to a degree dependent on the liquid and the withdrawal rate. We are engaged in NASA-supported work to optimize the characteristics of test-gratings made using this process and to measure their EUV efficiency after application of multilayers. The gratings have a density of 3600 grooves $\mathrm{mm}^{-1}$ and blaze angles between $2^{\circ}$ and $4^{\circ}$. Theoretical models produced using AFM-measured groove profiles from these test-gratings yield groove efficiencies approaching $66 \%, 50 \%$, and $30 \%$ in the first, second, and third orders, respectively. Further studies to improve the groove profile are under way at Zeiss, and will be completed in 2003.

From these results it is clear that multilayer-grating technology has reached a mature state, and predictions of effective area can be regarded as reasonably accurate. Using a MS-EXCEL spreadsheet we calculated the effective area of each $A P E X$ spectrometer as the simple product of the 6 factors discussed above, thus invoking the scalar assumption. For a few test cases we verified the validity of this assumption using the software package PC Grate $2000 \mathrm{MLX}$, which is one of the latest in a series of grating modeling programs available from International Intellectual Group (IIG), Inc. This program uses a modified integral method, and we have used earlier versions of it in calculating the efficiency of a bare and coated gratings. ${ }^{17,19,20,23}$ In all tests good agreement was found between the model and the simple product of groove efficiency and multilayer reflectance.

Table 2 shows parameter values for the APEX baseline configuration, where each of the 8 color-coded columns corresponds to one of the spectrometers shown in Fig. 3. Black-background row labels identify the spectrometer number and its peak design wavelength. The 8 peak wavelengths were chosen to span the range 90-290 $\AA$ somewhat uniformly and to be located near strong lines of astrophysical interest. ${ }^{1}$ In the science context (section 1), the shortest two wavebands are most important for CVs and extragalactic studies, the middle four wavebands for stellar coronae studies (designed for the highest resolution), and the longest two or three wavebands for WD studies. Of course, all wavebands are pertinent to ISM studies. White- and tan-background row labels indicate input quantities, while pink- and gray-background row labels are derived quantities.

The uppermost section of rows gives basic grating properties such as radius, groove density, and order. To maximize efficiency all spectrometer orders are positive (inside) so that the diffraction angle $U$ is zero (diffracted order lies on the grating normal). To simplify instrument mechanical design we have varied the grating groove density between spectrometers as the peak wavelength and order vary so that the angle of incidence $U$ and consequently the resolving power are held constant at one of two values $\left(=4.13^{\circ}\right.$ or $5.45^{\circ} ; R=8640$ or 11400$)$. Multilayer reflectance for each waveband was calculated over a wide range in wavelength. However, practical limitations on MCP detector size truncate this range, and wavelength 
limits are indicated in the middle rows of Table 2. We have assumed an active detector size of $50 \mathrm{~mm}$ in the dispersion direction, which is a comfortable value with present MCPs. (In practice spacecraft motion will require a slightly large size.)

Table 2. Baseline $A P E X$ configuration: Balance Wavelength Coverage and Peak Effective Area

\begin{tabular}{|c|c|c|c|c|c|c|c|c|}
\hline Spectrometer & 1 & 2 & 3 & 4 & 5 & 6 & 7 & 8 \\
\hline Grating Radius (mm) & 3000 & 3000 & 3000 & 3000 & 3000 & 3000 & 3000 & 3000 \\
\hline Detector+Grating Resolution (microns) & 25 & 25 & 25 & 25 & 25 & 25 & 25 & 25 \\
\hline grooves $/ \mathrm{mm}$ & 3600 & 3000 & 3600 & $3170^{\circ}$ & 2715 & 3600 & 3200 & 2940 \\
\hline Order & 2 & 2 & 2 & -2 & $2^{-}$ & 1 & 1 & 1 \\
\hline - angle of incidence (degrees). & 4.13 & 4.13 & 5.45 & 5.46 & -5.45 & 4.13 & 4.13 & 4.13 \\
\hline - Resotving power & 8640 & 8640 & 11405 & $11 \sqrt{2}$ & 17,03 & 8640 & 8640 & 8644 \\
\hline Peak Wavelength (Angstroms) & 100 & 120 & 132 & 150 & 175 & 200 & 225 & 245 \\
\hline Detector Active Size $(\mathrm{mm})$ & 50 & 50 & 50 & 50 & 50 & 50 & 50 & 50 \\
\hline Wavelength Range (Angstroms) & 23.1 & 27.8 & 23.1 & 26.3 & 30.7 & 46.3 & 52.1 & 56.7 \\
\hline Lower Limit (Añstroms) & 88.4 & 106.1 & 120.4 & 136.9 & 150.7 & 176.9 & 199.0 & 216.7 \\
\hline Upper Limit (Āngstroms) & 111.6 & 133.9 & 143.6 & 163.1 & $7903^{-}$ & 223.1 & 251.0 & 273.3 \\
\hline \# periods Multilayer (nodal/spacer) & $19 \mathrm{~B} / \mathrm{Rh}$ & 12 Be/Mo4Ru6 & $12 \mathrm{Mo} 2 \mathrm{C} / \mathrm{Si}$ & $10 \mathrm{MozCISI}$ & 6 M02019I & 9 Mo2CiSi & $8 \mathrm{Mo} 2 \mathrm{C} / \mathrm{Si}$ & $7 \mathrm{Mo} 2 \mathrm{C} / \mathrm{Si}$ \\
\hline \%Saturation & 48.1 & 48.1 & 53.4 & {$[-58.1-0$} & $-30.7-$ & -79.2 & 81.9 & 81.7 \\
\hline Multilayer Peak Reflectance & 0.307 & 0.361 & 0.388 & 0.382 & 0.287 & 0.383 & 0.341 & 0.308 \\
\hline$T_{\text {spacor (Angstroms) }}$ & 25.26 & 30.35 & 35.10 & 41.75 & 50.97 & 67.93 & 81.51 & 91.29 \\
\hline$T_{\text {nodal }}$ (Angstroms) & 26.61 & 31.87 & 33.40 & 37.16 & $4 \overline{2} \overline{92}$ & 38.57 & 39.10 & 40.46 \\
\hline Detector Quantum Efficiency & 0.55 & 0.50 & 0.45 & 0.42 & 0.40 & 0.30 & 0.30 & 0.30 \\
\hline Filter Transmission & 0.85 & 0.78 & 0.74 & 0.68 & 0.55 & 0.50 & 0.44 & 0.40 \\
\hline Groove Efficiency & 0.50 & 0.50 & 0.50 & 0.50 & 6.50 & 0.50 & 0.50 & 0.50 \\
\hline Collimator Transmission & 0.90 & 0.90 & 0.90 & $0.90^{-}$ & 60 & 0.90 & 0.90 & 0.90 \\
\hline Grating Diameter $(\mathrm{cm})$ & 34 & 34 & 34 & 34 & 34 & 34 & 34 & 34 \\
\hline Geometrical Area $\left(\mathrm{cm}^{\wedge} 2\right)$ & 907.9 & 907.9 & 907.9 & 907.9 & $907.9^{-}$ & 907.9 & 907.9 & 907.9 \\
\hline Integral (Angstroms cm²) & 315.7 & 541.5 & 551.3 & 628.9 & 5926 & 559.7 & 552.3 & 554.4 \\
\hline Plenath coverade & & & & & & & & \\
\hline elengin coverage & 10 & $\mathrm{~cm}^{\wedge} 2=$ & 150.7 & Angstroms & 2 Integrais & (Angstrom & $\left.3 \mathrm{~cm}^{n} 2\right)=$ & 4296.6 \\
\hline
\end{tabular}

We calculated multilayer reflectance profiles using the number of periods, the materials, and the thickness values for the nodal and spacer layers given in Table 2. The ratio of the nodal material thickness to the period thickness $($ was optimized for the number of periods. The reflectance profiles were calculated using an in-house program, and optical constants were derived from a number of sources in the literature (e.g., ref. 29 and further updates). Cross-calibration of the results of this program with those from other models such as the PC Grate program and the online software at the Center for X-ray Optics website produces excellent agreement.

A simple two-material design was assumed for all wavebands. In practice some diffusion of materials occurs, and this coupled with finite interface roughness reduces reflectance profiles by a small amount, typically a factor of $\sim 0.9$ at $100-300$ $\AA$. For example, a diffusion layer of $\mathrm{Mo}_{\mathrm{x}} \mathrm{Si}_{\mathrm{y}}$ can be as thick as $15 \AA$, a significant fraction of the period, especially at shorter wavelengths. The effects of interface roughness are smaller because multilayers tend to propagate the substrate roughness, and ion-etched gratings have roughness in the range 3-5 $\AA$ rms. However, more complex multilayer designs can mitigate the effects of both diffusion and roughness, ${ }^{23}$ and thus the two-material designs presented here are reasonable estimates. With increasing number of periods deposited, the multilayer bandpass will narrow, but the peak reflectance will grow until it reaches some "saturation" value, at which point the incident radiation no longer reaches the underlying substrate. The integral coverage attains a shallow peak at some number of periods in between 1 period and the number at saturation. For the $A P E X$ baseline configuration, we have chosen to balance peak effective area against wavelength coverage, and therefore the number of periods used is at or near this shallow maximum in integral coverage. The peak reflectance produced by the calculation is shown in Table 2 as well as its ratio to the saturation peak reflectance (\%Saturation). We have chosen $\mathrm{Mo}_{2} \mathrm{C} / \mathrm{Si}$ for the 6 spectrometers with peak wavelengths longer than the Si L-edge at $\sim 124 \AA$. For shorter wavelengths other materials must be used, and we have chosen $\mathrm{B} / \mathrm{Rh}$ and $\mathrm{Be} / \mathrm{Mo}_{4} \mathrm{Ru}_{6}$ for the $100-\AA$ and $120-\AA$ wavebands, respectively. We do not claim that these are the only materials possible or even the best. However, $\mathrm{Mo} / \mathrm{Si}$ and its variants have been the standard at long wavelengths for many years, and much expertise has been gained, so that fabricated saturated multilayers are produced regularly with reflectances within $95 \%$ of theoretical limits. Diffusion and fabrication problems may occur with other materials, especially at the shorter wavelengths. However, the choices made here are based on our current experience.

Table 2 illustrates some general properties of multilayers. First, multilayers function as synthetic Bragg crystals with the period thickness an integral number times half the peak wavelength. Hence the nodal and spacer layer thickness increases 
with wavelength. Second, the number of periods required for saturation and consequently our non-saturated choices decreases with increasing wavelength. Third, the optical constants of materials are such that multilayers attain their peak performance at $\sim 100-130 \AA$.

The multilayer reflectance profile dominates the variation in $\mathrm{A}_{\mathrm{eff}}$ over any spectrometer waveband, and therefore filter transmission, groove efficiency, collimator transmission, detector $\mathrm{QE}$, and of course grating area were held constant in each spectrometer waveband at the value determined for the peak multilayer wavelength (Table 2). The multilayer reflectance profiles were multiplied by these quantities to produce $A_{\text {eff }}$ curves for each spectrometer. This approximation, which can be refined later to include wavelength variation in $\mathrm{QE}$ and filter transmission, is good enough for our purposes here, namely to obtain general knowledge of the $A P E X \mathrm{~A}_{\text {eff }}$ and how it varies with parameters.

The bottom rows in Table 2 contain three metrics, which are useful in comparing spectrometer performance of alternate configurations. For each spectrometer we calculate the Integral $\left(A_{\text {eff }} \times\right.$ Wavelength) over the waveband as limited by detector size. The total Integral sum for all spectrometers is also given in the lower right corner. Finally, we calculate the total wavelength coverage at $A_{\text {eff }}$ greater than the cutoff value of $10 \mathrm{~cm}^{2}$. This value of $150.7 \AA$ is about $75 \%$ of the range 90 $290 \AA$.

Finally, although Table 2 was primarily used for calculating $\mathrm{A}_{\text {eff }}$, we also calculated a nominal spectrometer resolving power using the simple equation $R=f \sin \left(J / x\right.$, where $f$ is the focal length, _ is the angle of incidence, and $\_x$ is the quadratic sum of the detector resolution and the grating aberrations. We have assumed $25 \mathrm{~m}$ for this sum, which is an approximation. A more rigorous analysis involving ray-tracing is discussed in section 6; however, this simple approximation is useful in quickly comparing different $A P E X$ configurations.

Fig. 4 shows the $\mathrm{A}_{\text {eff }}$ curves for the APEX baseline configuration. The 8 spectrometer wavebands overlap, and the black line gives the composite result. Discontinuities in the black line are caused by the waveband cutoffs produced by the finite detector size, and therefore should cause no concerns for calibration difficulties.

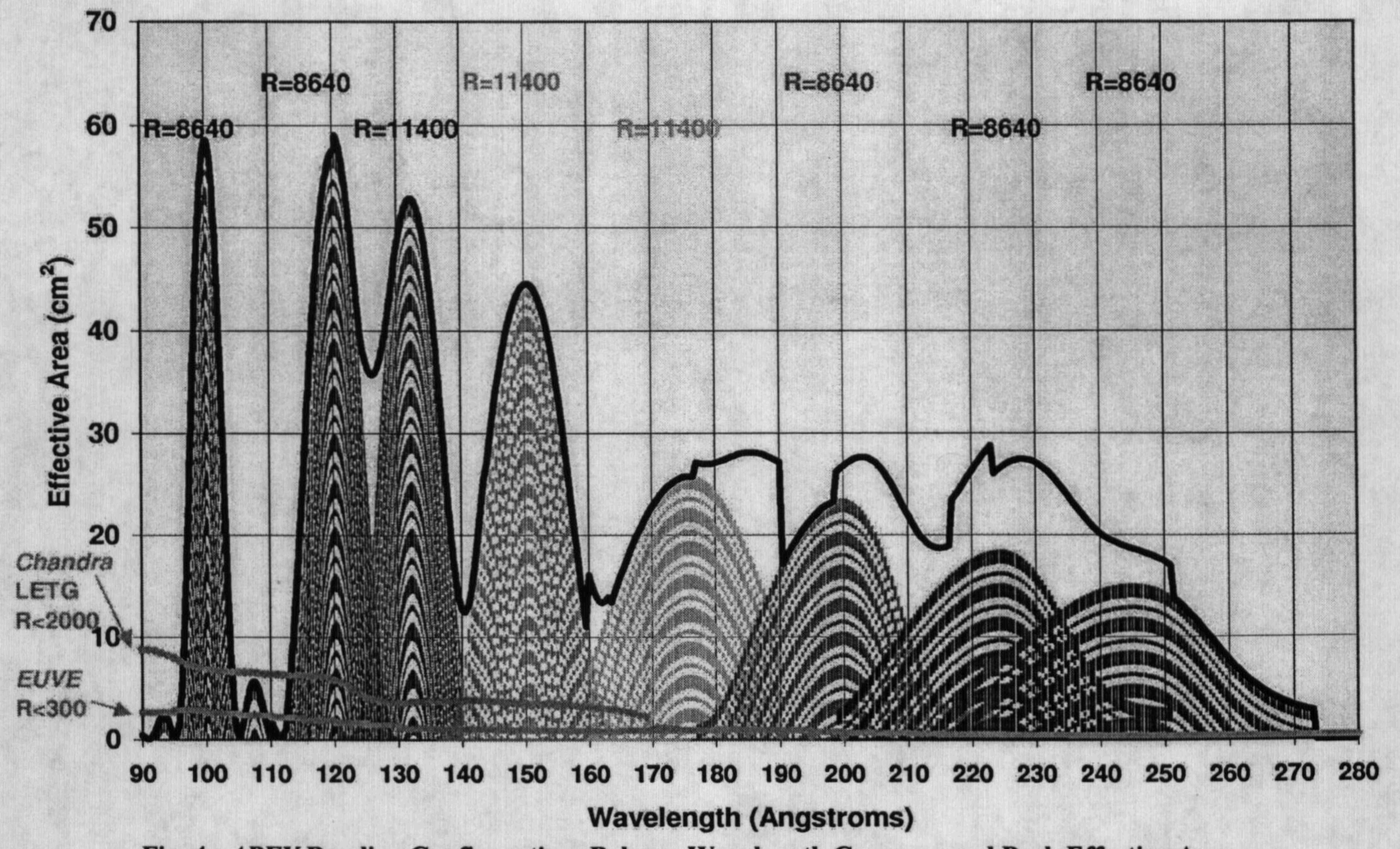

Fig. 4. APEX Baseline Configuration: Balance Wavelength Coverage and Peak Effective Area. 
Also shown in Fig. 4 is the $\mathrm{A}_{\text {eff }}$ for the sum of the first orders with the Chandra Low Energy Transmission Grating (LETG) as well as the composite $\mathrm{A}_{\text {eff }}$ for the three $E U V E$ spectrometers. $A P E X$ will achieve peak effective areas of $50-60 \mathrm{~cm}^{2}$ at selected wavelengths, but with coverage exceeding $10 \mathrm{~cm}^{2}$ over $75 \%$ of the range $90-290 \AA$. The peak values exceed the EUVE and Chandra values by an order of magnitude or more everywhere in the region of overlap, and the Chandra LETG response extends only to $\sim 170 \AA$. More importantly, the $A P E X$ resolving power exceeds that of Chandra by at least a factor of 5 , and $E U V E$ by a factor of $\sim 30$. This combination of simultaneous high effective area and resolving power makes $A P E X$ an ideal instrument to open a new door in EUV spectroscopy.

\section{EFFECTIVE AREA FOR ALTERNATE APEX CONFIGURATIONS}

We constructed the spreadsheet to investigate quickly how different configurations affect the $A P E X \mathrm{~A}_{\text {eff }}$. One of the simplest changes is to deposit enough periods to saturate the multilayers, keeping all other parameters the same as in the baseline case. The results are shown in Fig. 5 and Table 3. The peaks double to $\sim 120 \mathrm{~cm}^{2}$ at $100-120 \AA$ wavelengths (note the factor of 2 scale change in the ordinate as compared to Fig. 4), but the gain become negligible at longer wavelengths. However, this configuration might be useful if it was decided that monitoring fewer spectral lines with greater sensitivity (perhaps for better temporal resolution) was more desirable than wavelength coverage. Note that the Integral metric for the short wavelength spectrometers increases slightly compared to the results in Table 2, while that for the long wavelength spectrometers decreases in proportion so that the total Integral sum remains approximately constant. It is interesting that the wavelength coverage decreases by only one-sixth to a value of $126.8 \AA$.

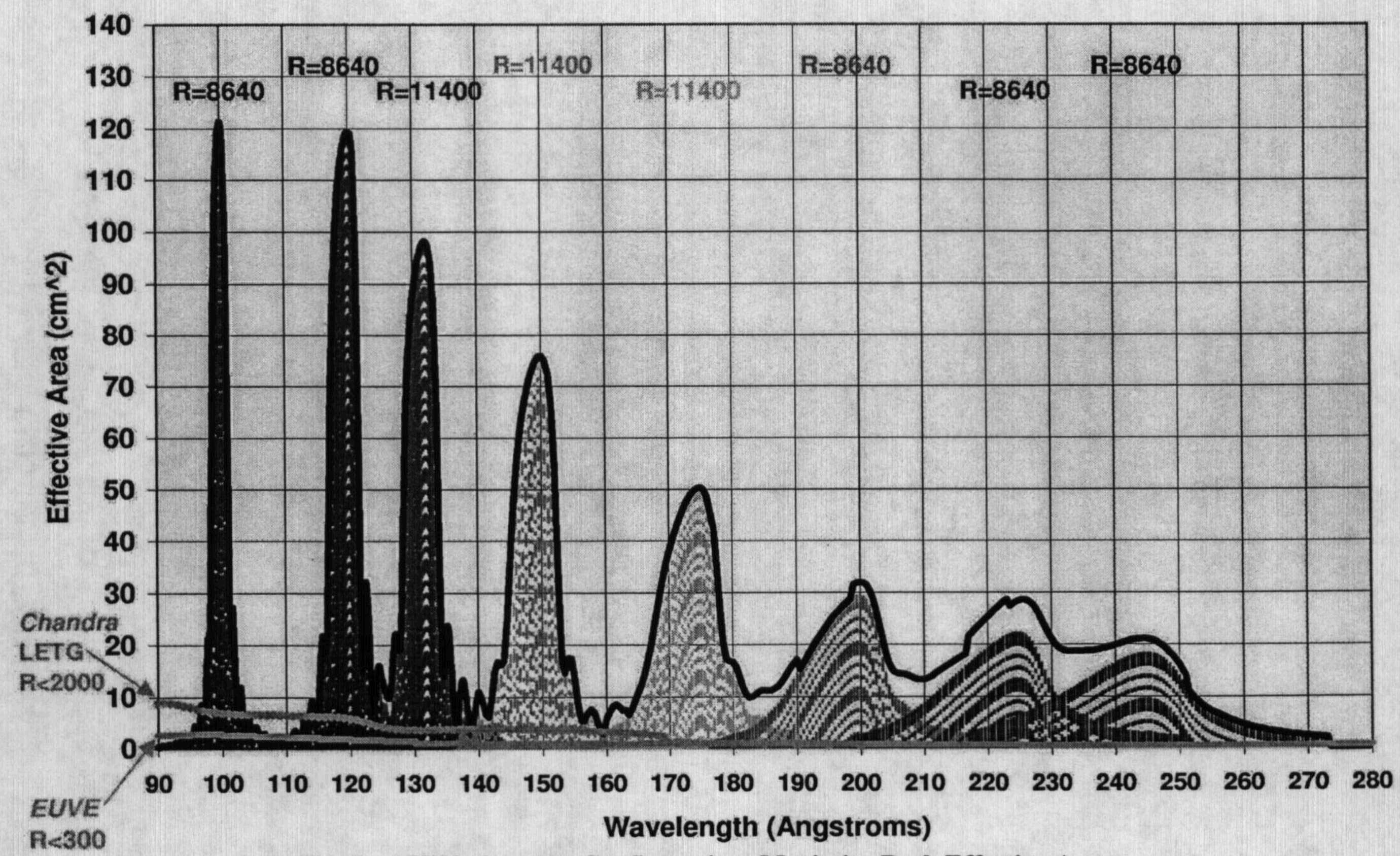

Fig. 5. APEX Alternate Configuration: Maximize Peak Effective Area.

Table 3. Alternate $A P E X$ configuration: Maximize Peak Effective Area

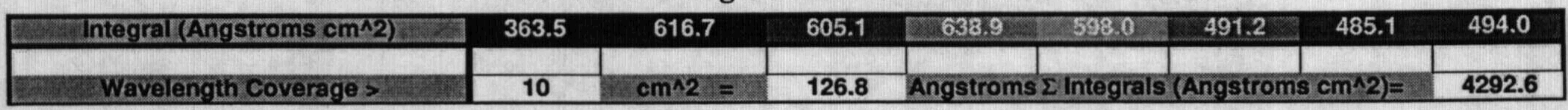


Of course hybrid alternates of saturated and non-saturated multilayers are also possible. We examined several other configurations. First, to increase wavelength coverage over $10 \mathrm{~cm}^{2}$, we reduced the number of multilayer periods on the 4 shortest wavelength spectrometers so that the summed $A_{\text {eff }}$ (black line) was approximately constant at $20-30 \mathrm{~cm}^{2}$. However, the gain in wavelength coverage was marginal $(154 \AA)$, making this alternative unappealing. Second we replaced the multilayers with single layers of gold on all spectrometers. $\mathrm{A}_{\mathrm{eff}}$ dropped to values less than or equal to the EUVE values in Figs. 4 and 5 with no coverage over $10 \mathrm{~cm}^{2}$, dramatically demonstrating the gain imparted by the multilayer coatings. Third, we varied the detector size between $40 \mathrm{~mm}$ and infinity, and we were surprised to discover that strenuous efforts to increase detector size and thereby capture more of the each spectrum were unwarranted. The gain with no detector limit was marginal (153 $\AA$ wavelength coverage above $10 \mathrm{~cm}^{2}$ and a total Integral sum of $4630 \AA \mathrm{cm}^{2}$ ). In summary, these simple tests showed that even without refining the calculations we could already judge the effects of major design changes on $\mathrm{A}_{\text {eff }}$.

However, with a limited number of spectrometers available it is obvious that the biggest arguments will come over placement of wavebands. There is considerable freedom in each selection as it depends solely on multilayer thickness, and small changes can be accommodated easily. In practice the selections would be driven by the scientific objectives as determined by an APEX Science Working Group (SWG). Therefore, the SWG can use the spreadsheet as a tool for refining its goals. An objective method might be to weight the scientific value of spectral lines to optimize waveband locations and widths.

One argument that has been raised consistently against EUV instruments is that the transparency of the ISM limits their use to primarily local galactic observations. For at least 2 reasons, this is something of a misperception. First, transparency is a steep function of wavelength. Fig. 6 shows for three different hydrogen densities the distance at which incident radiation is attenuated $90 \%$, as a function of wavelength. ${ }^{30}$ The galaxy becomes more transparent at wavelengths approaching $100 \AA$, and therefore short wavelengths would allow for a broad range of galactic and extragalactic observations. Second the ISM is patchy, and in Fig. 7 we show the accessible space out to $20 \mathrm{pc}$ as a function of wavelength and of galactic coordinates. Coverage is large and although it decreases rapidly for larger distances, holes (e.g., the Lockman hole) provide for a reasonable solid angle for extragalactic work. Finally, APEX is not a survey instrument and most likely its targets will be chosen carefully from the present list of EUV sources $(1000)$ provided by the EUVE and the ROSAT WFC all-sky surveys.

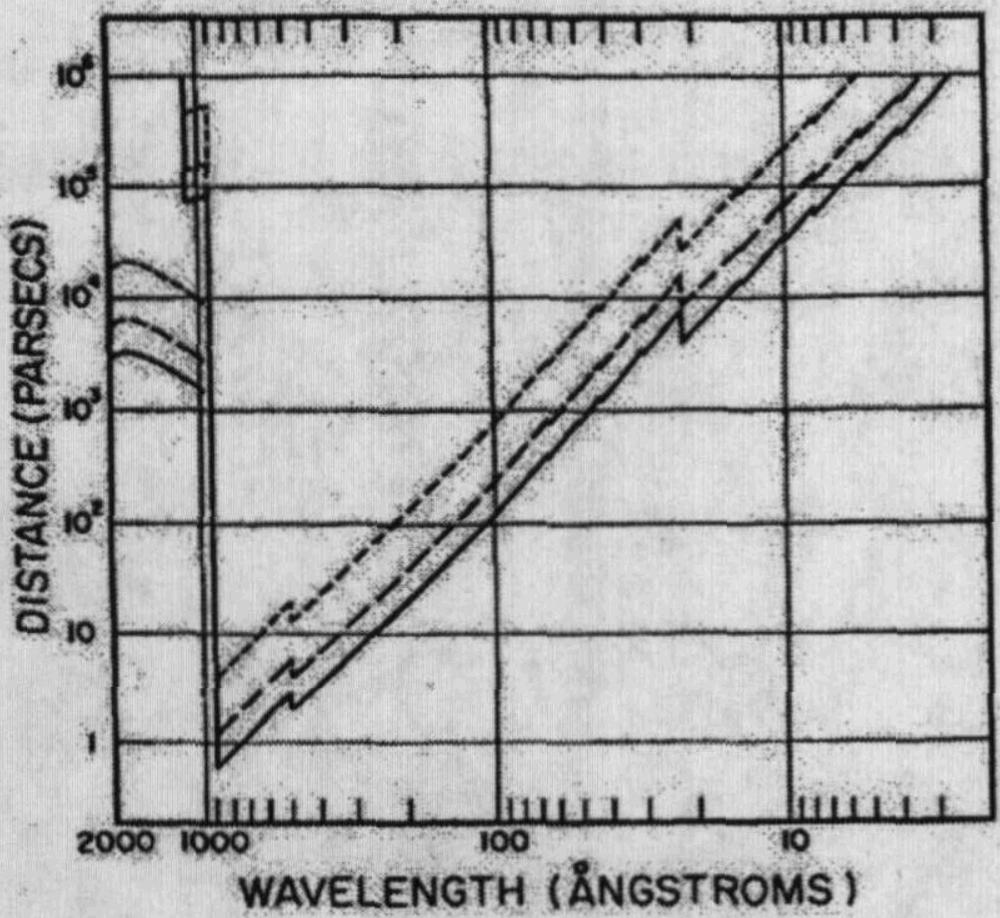

Fig. 6. Distance at which incident radiation is attenuated $90 \%$. An un-ionized ISM of normal composition is assumed with $n_{\mathrm{H}}=0.2,0.1$, and $0.03 \mathrm{~cm}^{-3}$, for solid-, long-, and short-dashed curves, respectively.

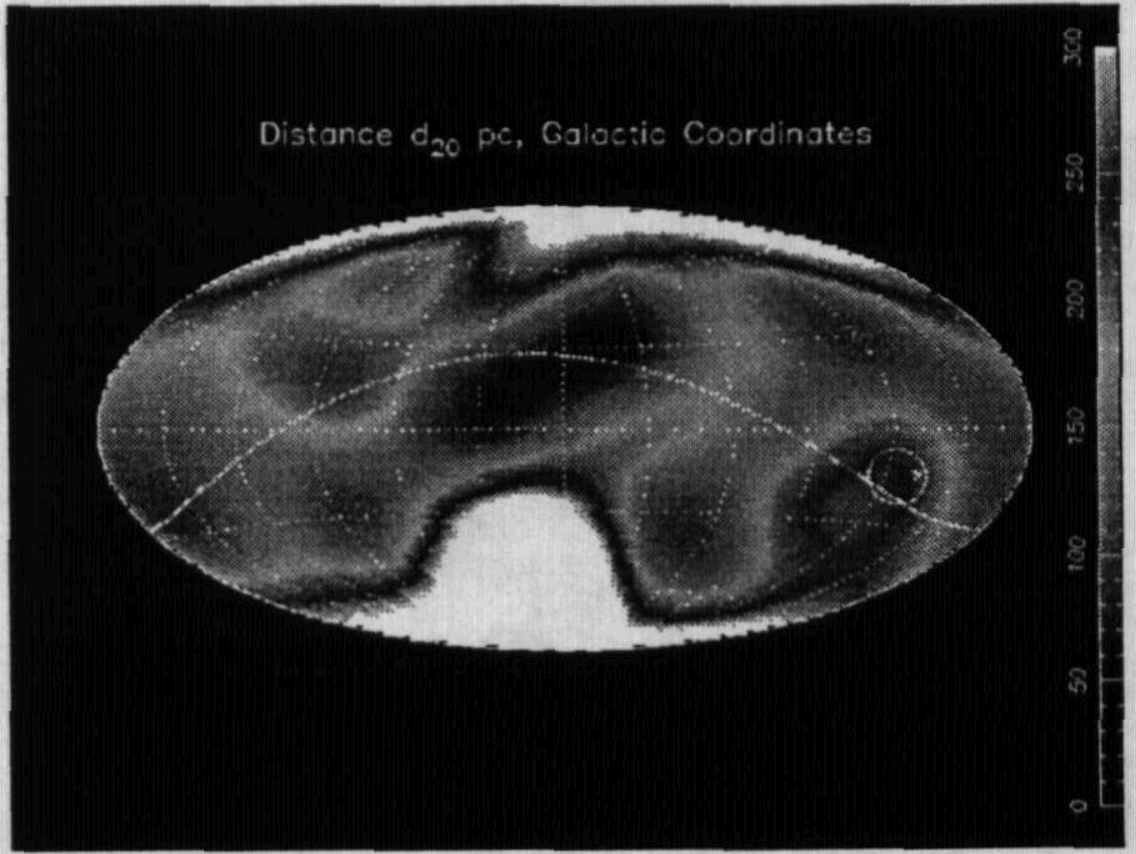

Fig. 7. Accessible space out to 20 pc as a function of galactic coordinates and of wavelength ( $\AA$; see color legend)

Nevertheless, we have used the $A P E X$ effective area spreadsheet to construct a radically different configuration that is optimized for wavelengths less than $100 \AA$. The results are shown in Fig. 8, where the wavebands are spaced in 5- $\AA$ interval. The configuration balances wavelength coverage with peak effective area for the 6 longest wavebands, but multilayers for the 2 shortest wavelength spectrometers are saturated. Because it has favorable optical constants, a B/Rh multilayer was chosen for these calculations; however, fabrication issues might make other materials such as W/B ${ }_{4} \mathrm{C}$ more practical. The 5 shortest wavelength spectrometers operate in the third grating order, and we have assumed a groove efficiency of $30 \%$ to reflect current predictions, resulting in a sharp drop in peak $A_{\text {eff }}$ as compared to the longer waveband spectrometers. Although the 
peak vaues of $\mathrm{A}_{\text {eff }}$ are comparable to those in the APEX baseline configuration (Fig. 4), the wavelength coverage greater than $10 \mathrm{~cm}^{2}$ is only $22.1 \AA$, about half of the range. The total Integral sum is only $741.3 \AA \mathrm{cm}^{2}$, about a sixth of the result for the $A P E X$ baseline configuration. Moreover, the Chandra LETG exceeds the peak $A P E X \mathrm{~A}_{\text {eff }}$ at wavelengths shorter than $70 \AA$, although the $A P E X$ resolution is still better. Thus, any use of wavebands shorter than $\sim 100 \AA$ in APEX will require judicious choices among science tradeoffs. However, the use of such wavebands in future larger instruments should not be rejected outright, as cosmological objects will large distances will have important spectral lines redshifted to EUV wavebands.

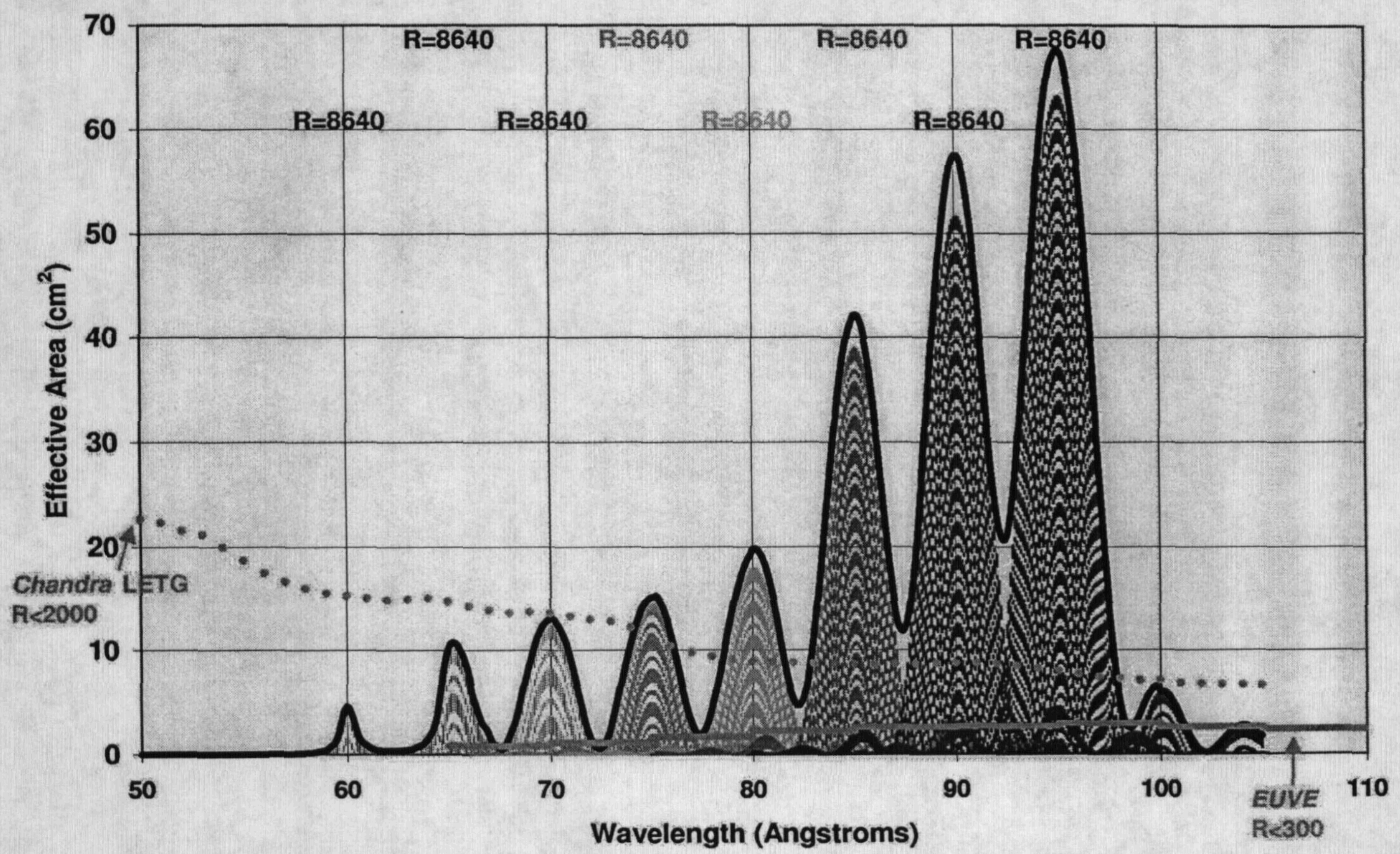

Fig. 8. APEX Alternate Configuration: Balance Wavelength and Effective Area at Short Wavelengths

Finally, we calculated $A_{\text {eff }}$ curves for a few selected wavebands at wavelengths longer than $300 \AA$. Although $A_{\text {eff }}$ drops off with increasing wavelength, reasonable observations might be attempted on a number of relatively nearby hot stars with low ISM absorption, for example the giant $\mathrm{B}$ stars $\mathrm{CMa}$ and $\mathrm{CMa}$, in which significant understanding can best be accomplished using high-resolution EUV spectra. ${ }^{31}$ Moreover, important and well-known helium transitions occur at 304 and $584 \AA$, and abundance measurements of ${ }^{3} \mathrm{He}$ in the local ISM are cosmologically important. ${ }^{32} \mathrm{With} \mathrm{Mo} / \mathrm{Si}$ and its variants, the results predict a peak $A_{\text {eff }}$ of $\sim 7 \mathrm{~cm}^{2}$ at $304 \AA$ decreasing to a peak of $\sim 2 \mathrm{~cm}^{2}$ at $\sim 500 \AA$, compared to $\sim 1 \mathrm{~cm}^{2}$ for single gold layers. Waveband FWHM increases gradually from 30 to $50 \AA$ over this range. However, the paucity of targets makes wavebands with peaks longer than $304 \AA$ unlikely candidates for $A P E X$, and such targets will probably have to wait until more suitable instruments can be designed.

\section{APEX RESOLUTION}

The nominal spectrometer resolution given in Figs. 4, 5, and 8 is based on a simple equation derived from focal length and groove density. However, a more rigorous analysis requires ray-tracing. We used the program BEAM FOUR from Stellar Software to create ray-tracing images for the $A P E X$ baseline configuration spectrometer that peaked at $175 \AA$. (Good agreement was found between results from this program and those from in-house software.) Two types of gratings were investigated, the first with a spherical figure and the second with a paraboloidal figure. In both cases, the gratings were mounted in an off-axis Wadsworth mount, with the detector normal going through the center of the grating. Monochromatic ray-tracing images were created at 11 wavelengths separated in $3-\AA$ intervals over the range $160-190 \AA$. Fig. 9 is a collage of 
ray-tracing images located only on the short wavelength side of the multilayer peak, as the images on the long wavelength side were symmetrical. At the multilayer peak wavelength the paraboloidal image has negligible size and is therefore much smaller than the image produced by the spherical grating, which exhibits so-called "spherical aberration" at all wavelengths. However, aberrations for the paraboloidal grating grow faster with off-axis angle and consequently wavelength than for the spherical grating, and the images have a higher degree of asymmetry. At the multilayer's extreme wavelengths as defined for a 50-mm detector (Table 2), paraboloidal and spherical gratings produce images of comparable size

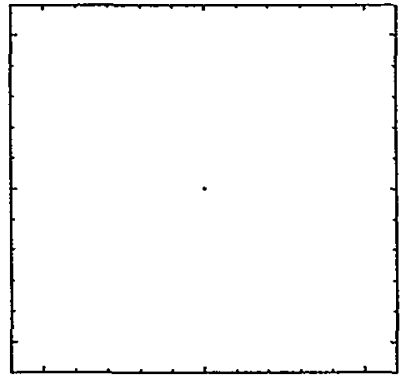

175 Angstroms

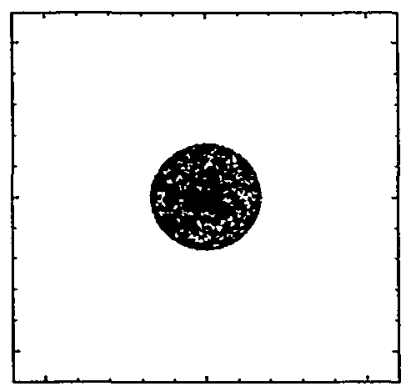

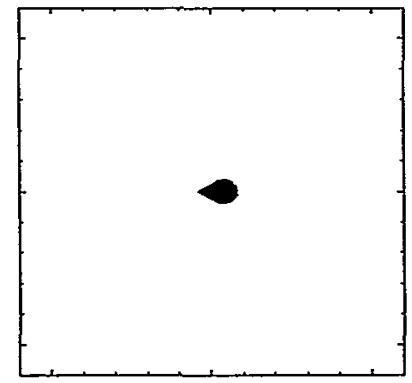

172 Angstroms

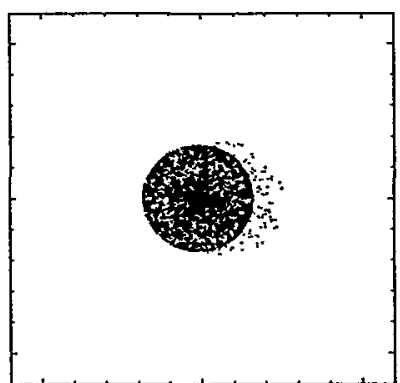

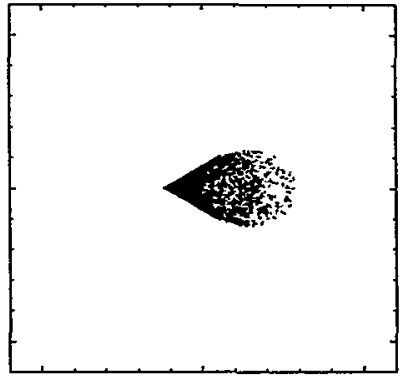

166 Angstroms

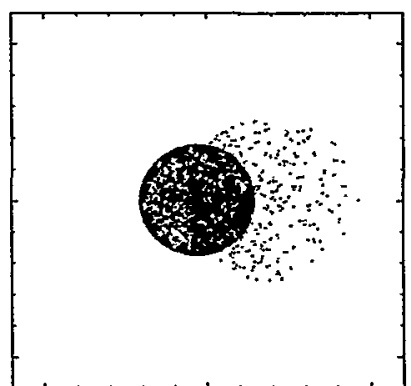

Fig. 9. Ray tracing images at selected wavelengths for a paraboloidal grating (top row) and a spherical grating (bottom row). The image frame size is $120 \_\mathrm{m}$.

We collapsed the data for each ray-tracing image into a histogram along the dispersion direction (horizontal in Fig. 9), determined the image width at $90 \%$ power, and used this value as a measure of grating aberrations. Although images show that grating aberrations are negligible near the multilayer peak wavelength, we conservatively chose a minimum value of 10 _ $m$ for grating aberrations whenever the histogram produced smaller values. These values were added in quadrature to the assumed detector spatial resolution $(15 \mathrm{~m})$. The resolution curve for the paraboloidal grating, shown in Fig. 10, achieves values near 16,000 at the peak multilayer wavelengths. It exceeds the curve of the spherical grating everywhere over the waveband and exhibits more variation with wavelength. For about half the waveband near its peak wavelength, the resolving power is greater than the nominal value of 11,400 (Table 2), but less than that at the extreme wavelengths. Thus the nominal resolving power calculated from the simple equation represents a reasonable average. Similar results were obtained from analyses of the $100-\AA$ and $245-\AA A P E X$ spectrometers. In conclusion, this preliminary exercise demonstrates that a paraboloidal figure is preferable to spherical one, and that the $A P E X$ resolution goals can be achieved.

Fig. 10. Resolution curves for paraboloidal and spherical gratings as derived from raytraced images.

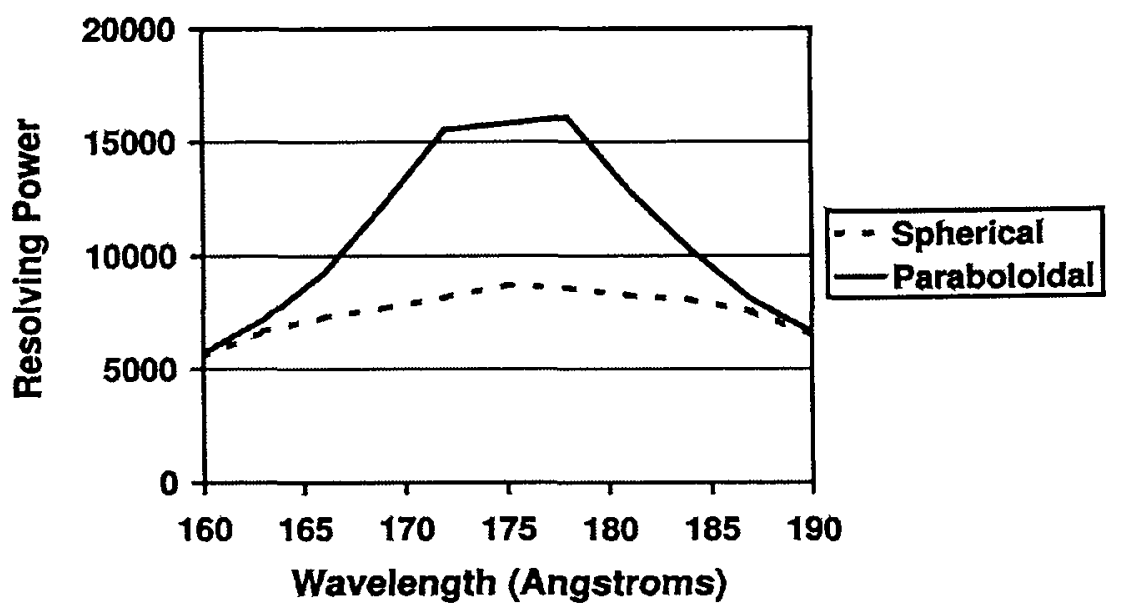




\section{CONCLUSIONS}

We have presented a practical design for a SMEX satellite instrument, $A P E X$, which could be launched by a Taurus booster. $A P E X$ consists of a suite of 8 spectrometers, each employing a multilayer-coated diffraction grating operating at near-normal incidence and a high-resolution MCP detector. A baseline configuration of $A P E X$ would achieve peak $\mathrm{A}_{\text {eff }}$ of $30-60 \mathrm{~cm}^{2}$ at selected wavelengths in the range $90-290 \AA$, with $75 \%$ of this range having $A_{\text {eff }}$ greater than $10 \mathrm{~cm}^{2}$. The average resolving power would be 8640-11400. Variations on the baseline design are also possible, which allow for optimizing the design for a given set of spectral features and for extending or shifting coverage to shorter and/or longer wavelengths. APEX would be capable of a wide range of science, particularly in studies of stellar coronae and flares, evolution of WDs, accretion regions in CVs and AGNs, and the nature of the ISM. However, the exact mix of science objectives has yet to be determined. Nevertheless it is clear that high-resolution astronomical spectroscopy, which allows the identification of weak emission lines, the measurement of Doppler shifts and line profiles, and the detection of narrow absorption features, leads to a complete understanding of the density, temperature, abundance, magnetic, and dynamic structure of astrophysical plasmas. Observations at EUV wavelengths are critical to understanding plasmas with temperatures in the range $10^{5}-10^{8} \mathrm{~K}$.

The technological and theoretical experience in high-reflectance multilayers and ultrasmooth diffraction gratings of accurate groove profile has been developed in over a decade of research so that efficiencies approaching theoretical limits are now being obtained. This maturity provides $A P E X$ with a wide design margin, with little technical and consequently programmatic risk involved.

\section{ACKNOWLEDGEMENTS}

This work was supported by the Office of Naval Research under W.U. 3641: Application of Multilayer Coated Optics to Remote Sensing, by the Naval Research Laboratory Bid and Proposal Job Number 76-6039, by NASA-Defense Purchase Request S-47440F: A Study of the Physics and Composition of White Dwarf Atmospheres using High Resolution EUV Spectroscopy, and by NASA-Defense Purchase Request W-19,881: Liquid-Overcoated Blazed Gratings.

\section{REFERENCES}

1. U. Feldman, P. Mandelbaum, J. F. Seely, G. A. Doschek, and H. Gursky, "The potential for plasma diagnostics from stellar extreme-ultraviolet observations," Ap.J. Suppl. 81, 387-408 (1992).

2. M. A. Barstow, N. P. Bannister, G. W. Fraser, R. G. Cruddace, M. P. Kowalski, G. G. Fritz, D. J. Yentis, H. Gursky, J. S. Lapington, J. A. Tandy, B. Sanderson, T. W. Barbee, Jr., W. H. Goldstein, and J. F. Kordas, "J-PEX: The Joint Astrophysical Plasmadynamic Experiment," $11^{\text {th }}$ European Workshop on White Dwarfs, University of Tromso, Tromso, Norway, 29 June-3 July 1998.

3. R. G. Cruddace, M. P, Kowalski, G. G. Fritz, D. J. Yentis, H. Gursky, M. A. Barstow, N. P. Bannister, G. W. Fraser, J. S. Lapington, J. A. Tandy, B. Sanderson, T. W. Barbee, Jr., W. H. Goldstein, and J. F. Kordas, "High Resolution Astrophysical Spectroscopy," Workshop on Small Missions for Energetic Astrophysics: Ultraviolet Through Gamma Ray, Los Alamos National Laboratory, Los Alamos, New Mexico, 22-26 February 1999.

4. M. Kowalski, "Space Science Division's J-PEX instrument to provide new data on the evolution of white dwarf stars," NRL Labstracts (Naval Research Laboratory, Washington, D.C., 2001).

5. R. G. Cruddace, M. P. Kowalski, D. Yentis, C. M. Brown, H. Gursky, M. A. Barstow, N. P. Bannister, G. W. Fraser, J. E. Spragg, J. S. Lapington, J. A. Tandy, B. Sanderson, J. L. Culhane, T. W. Barbee, J. F. Kordas, W. Goldstein, and G. G. Fritz, "High-Resolution spectroscopy of G191-B2B in the extreme ultraviolet," Ap. J. Lett. 565, L47-L50 (2002).

6. M. A. Barstow, I. Hubeny, and J. B. Holberg, "Evidence for the stratification of Fe in the photosphere of G191-B2B," MNRAS 307, 884-894 (1999).

7. M. A. Barstow, I. Hubeny, and J. B. Holberg, "The effect of photospheric heavy elements on the hot DA white dwarf temperature scale," MNRAS 299, 520-534 (1998).

8. M. A. Barstow, N. P. Bannister, R. G. Cruddace, M. P. Kowalski, K. S. Wood, D. J. Yentis, H,. Gursky, T. W. Barbee, Jr., W. H. Goldstein, J. F. Kordas, G. G. Fritz, J. L. Culhane, and J. S. Lapington, "The Joint astrophysical Plasmadynamic Experiment (J-PEX): A high-resolution rocket spectrometer," Proc. SPIE 4854, this volume (2002).

9. C. M. Golembiewski, R. G. Cruddace, and M. P. Kowalski, "Collimator design for the J-PEX sounding rocket," Proc. SPIE 3445, 197-204 (1998).

This work was performed under the auspices of the U.S. Department of Energy by the University of California, Lawrence Livermore National Laboratory under Contract No. W-7405-Eng-48. 
10. O. H. W. Siegmund, "Emerging sensor technologies for future extreme ultraviolet astronomy missions," $200^{\text {th }}$ Meeting AAS, Topical Session "The Future of EUV Astronomy", NRL Memorandum Rep. in preparation (Naval Research Laboratory, Washington, DC 2002).

11. J. S. Lapington, and B. Sanderson, "Microchannel plate limited resolution with the vernier anode," Proc. SPIE 4139, 242-249 (2000).

12. M. P. Kowalski, J. F. Seely, R. G. Cruddace, J. C. Rife, C. M. Brown, G. A. Doschek, U. Feldman, T.W. Barbee, Jr., W. R. Hunter, G. E. Holland, and C. N. Boyer, "High-resolution EUV spectroscopy: performance of spherical multilayercoated gratings operated at near normal incidence," Proc. SPIE 1945, 164-175 (1993).

13. R. G. Cruddace, T. W. Barbee, Jr., J. C. Rife, and W. R. Hunter, "Measurements of the normal-incidence x-ray reflectance of a molybdenum-silicon multilayer deposited on a 2000-1/mm grating," Phys. Scr. 41, 396-399 (1990).

14. M. P. Kowalski, T. W. Barbee, Jr., R. G. Cruddace, J. F. Seely, J. C. Rife, and W. R. Hunter, "Efficiency and long-term stability of a multilayer-coated ion-etched holographic grating in the 125-133- $\AA$ wavelength region," Appl. Opt. 34, 7338-7346 (1995).

15. J. F. Seely, R. G. Cruddace, M. P. Kowalski, W. R. Hunter, T. W. Barbee, Jr., J. C. Rife, R. Eby, and K. G. Stolt, "Polarization and efficiency of a concave multilayer grating in the 135-250- $\AA$ region and in normal-incidence and SeyaNamioka mounts," Appl. Opt. 34, 7347-7354 (1995).

16. M. P. Kowalski, R. G. Cruddace, J. F., Seely, J. C. Rife, K. F. Heidemann, U. Heinzmann, U. Kleineberg, K. Osterried, D. Menke, and W. R. Hunter, "Efficiency of a multilayer-coated, ion-etched laminar holographic grating in the 14.516.0-nm wavelength region," Opt. Lett. 22, 834-836 (1997).

17. J. F. Seely, M. P. Kowalski, R. G. Cruddace, K. F. Heidemann, U. Heinzmann, U. Kleineberg, K. Osterried, D. Menke, J. C. Rife, and W. R. Hunter, "Multilayer-coated laminar grating with $16 \%$ normal-incidence efficiency in the $150-\AA$ wavelength region," Appl. Opt. 36, 8206-8213 (1997).

18. K. H. Hellwege, “_ber rasterf_mige Reflexionsgitter,” Z. Phys. 106, 6453-6458 (1937).

19. M. P. Kowalski, J. F. Seely, L. I. Goray, W. R. Hunter, and J. C. Rife, "Comparison of the calculated and the measured efficiencies of a normal-incidence grating in the 125-225- $\AA$ wavelength range," Appl. Opt. 36, 8939-8943 (1997).

20. W. R. Hunter, M. P.Kowalski, and R. G. Cruddace, "Investigation of the properties of an ion-etched plane laminar holographic grating," Appl. Opt. 40, 6157-6165 (2001).

21. M. P. Kowalski, J. F. Seely, W. R. Hunter, J. C. Rife, T. W. Barbee, Jr., G. E. Holland, C. N. Boyer, C. M. Brown, and R. G. Cruddace, "Dual waveband operation of a multilayer-coated diffraction grating in the soft X-ray range at nearnormal incidence," Appl. Opt. 32, 2422-2425 (1993).

22. M. P. Kowalski, T. W. Barbee, Jr., K. F. Heidemann, H. Gursky, J. C. Rife, W. R. Hunter, G. G. Fritz, and R. G. Cruddace, "Efficiency calibration of the first multilayer-coated holographic ion-etched flight grating for a sounding rocket high-resolution spectrometer," Appl. Opt. 38, 6487-6493 (1999).

23. M. P. Kowalski, H. Gursky, J. C. Rife, D. J. Yentis, R. G. Cruddace, T. W. Barbee, Jr., W. H. Goldstein, J. F. Kordas, K F. Heidemann, G. G. Fritz, W. R. Hunter, M. A. Barstow, G. W. Fraser, N. P. Bannister, J. S. Lapington, J. A. Tandy, and $B$. Sanderson, "Efficiency calibration of the four multilayer-coated holographic ion-etched flight gratings for sounding rocket spectrometer," Proc. SPIE 4498, 303-314 (2001).

24. J. C. Rife, W. R. Hunter, and R. T. Williams, "Features and initial performance tests of the grating/crystal monochromator," Nucl. Instrum. Methods, A246, 252-255 (1986).

25. J. C. Rife, H. R. Sadeghi, and W. R. Hunter, "Upgrades and recent performance of the grating/crystal monochromator," Rev. Sci. Instrum. 60, 2064-2067 (1989).

26. W. R. Hunter and J. C. Rife, "An ultrahigh vacuum reflectometer/goniometer for use with synchrotron radiation," Nucl. Instrum. Methods A246, 465-468 (1986).

27. M. P. Kowalski, R. G. Cruddace, J. F. Seely, J. C. Rife, and W. R. Hunter, "Uncertainties in reflectance measurements made on the NRL beam line X24C," NRL Memorandum Rep. 7620-95-7738 (Naval Research Laboratory, Washington, DC 1995).

28. K. Osterried, K. F. Heidemann, and B. Nelles, "Groove profile modification of blazed gratings by dip coating with hardenable liquids," Appl. Opt. 37, 8002-8007 (1998).

29. B. L. Henke, E. M. Gullikson, and J. C. Davis, "X-ray inteactions: photoabsorption, scattering, transmission, and reflection at $E=50-30,000 \mathrm{eV}, \mathrm{Z}=1-92$," At. Data Nucl. Data Tables, 54, 181-342 (1993).

30. R. G. Cruddace, F. Paresce, S. Bowyer, and M. Lampton, "On the opacity of the interstellar medium to ultrasoft X-rays and extreme ultraviolet radiaion," Ap.J. 187, 497-504 (1974).

31. J. Cassinelli, N. Miller, J. MacFarlane, D. Cohen, and N. Moeckel, "What can we learn from EUV studies of hot stars," $200^{\text {th }}$ Meeting AAS, Topical Session "The Future of EUV Astronomy", NRL Memorandum Rep. in preparation (Naval Research Laboratory, Washington, DC 2002). 
32. M. S. Turner, J. W. Truran, D. N. Shramm, and C. J. Copi, "Cosmological implications of the first measurement of the local ISM abundance of ${ }^{3} \mathrm{He}$," Ap.J. Lett. 466, L59-L62 (1996). 